\title{
EL ABORDAJE NUTRICIONAL EN EL TRATAMIENTO DE LA DEPRESIÓN
}

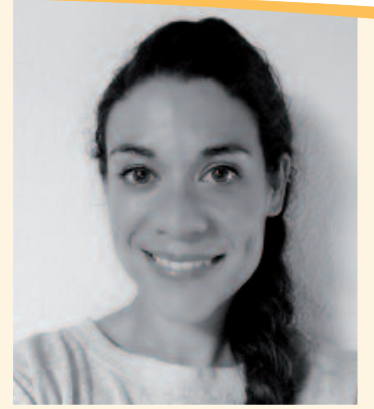

MIREIA LUGAR SANCHO

Dietista-nutricionista y psicóloga. Especialista del Área de Psiconutrición del Centro DINUT. Barcelona.

\section{INTRODUCCIÓN}

Según datos ofrecidos recientemente por la Organización Mundial de la Salud, 1 de cada 5 personas sufre actualmente depresión o ansiedad, llegando a ser de unos 300 millones la cifra de personas afectadas por depresión a nivel mundial ${ }^{1}$.

A pesar de que la mayoría de las teorías existentes sobre su etiología lo catalogan como un trastorno de origen multifactorial, durante décadas, los tratamientos de elección en el abordaje terapéutico de la depresión han sido los de tipo farmacológico y psicológico, dejando de lado la posibilidad de tratar otros factores modificables como los ambientales, el estilo de vida y los sociales²,3.

Este hecho, sumado a que la carga mundial de esta enfermedad sigue creciendo a una velocidad vertiginosa año tras año, ha propiciado por parte de la comu- nidad científica el cuestionamiento de la eficacia de dichos tratamientos, abriendo una puerta hacia otros enfoques terapéuticos conocidos como «tratamientos alternativos», en la búsqueda de otras vías que puedan asegurar una mayor adherencia a los tratamientos $y$, en consecuencia, un mayor éxito en los resultados.

El abordaje nutricional y la adopción de patrones de vida más saludables, como la realización de actividad física de forma regular, son actualmente una de las propuestas alternativas que suelen sugerirse con más frecuencia como coadyuvantes al tratamiento principal. La facilidad con la que pueden aplicarse por parte del paciente, tanto a lo que a coste económico como a realización se refiere, los mínimos - por no decir casi inexistentes - efectos adversos percibidos y una notable mejoría del estado de ánimo y percepción mayor de bienestar a corto plazo son algu-

Correspondencia: Mireia Lugar Sancho

Correo electrónico: mlugarsancho@gmail.com 
nos de los beneficios que abogan en su defensa.

\section{LA NUTRICIÓN Y SU IMPORTANCIA EN EL ABORDAJE TERAPÉUTICO}

Como en muchos procesos del cuerpo humano, la nutrición también desempeña en este caso un papel fundamental en la bioquímica y funcionamiento de dicha enfermedad. La bibliografía científica existente hasta el momento en el campo de la psiquiatría y la nutrición apunta hacia una relación directa entre el proceso inflamatorio y la depresión. Para ser más precisos, en los estudios realizados con pacientes afectados por este trastorno, se aprecia un aumento de los marcadores inflamatorios a nivel analítico, concretamente, en las citocinas. Estos marcadores serían, entre otros, los encargados de desarrollar toda la gama de síntomas depresivos ${ }^{4}$.

Además de esto, se ha apreciado de forma general que las personas afectadas por depresión desarrollan patrones alimentarios inadecuados, realizando dietas muy restrictivas y poco saludables, donde el descontrol en las comi- das y los alimentos procesados y ricos en grasas y azúcares predominan de forma destacable.

Por ello y con la certeza de que un seguimiento nutricional no puede más que aportar beneficios sobre la salud y el bienestar de los pacientes, cabe destacar finalmente que aquellas personas que siguen un programa dietético como herramienta de apoyo en un tratamiento multidisciplinario perciben una mejoría en su estado de ánimo, aumentando su motivación para retomar las riendas y el control de sus vidas, a la vez que suelen encontrarse anímica y físicamente mucho mejor.

\section{DIETA MEDITERRÁNEA Y ANTIINFLAMATORIA}

Teniendo en cuenta lo dicho anteriormente, la alimentación más adecuada en estos casos sería aquella que ayude a reducir y evitar la inflamación en el organismo, aparte de ser saludable, equilibrada y suficiente según las características individuales de cada paciente.

La dieta mediterránea, rica en verduras y fruta, cereales de grano entero, legumbres, aceite de oliva y pescado, se convierte en la opción más recomendada 5 .

Además de seguir las directrices de una dieta mediterránea, se recomienda de forma especial elevar el consumo de alimentos con potencial antiinflamatorio, así como realizar un consumo más reservado de alimentos ricos en grasas animales y azúcares, como la carne roja, azúcares refinados, alimentos procesados, etc. debido a que son ricos en nutrientes potencialmente inflamatorios.

El aporte regular en la dieta de los ácidos grasos del tipo omega3 resulta crucial en el enfoque terapéutico de las enfermedades que cursan con un proceso inflamatorio. El aumento en el organismo de las cantidades del ácido graso poliinsaturado eicosapentaenoico (EPA o ácido icosapentaenoico), perteneciente a la cadena de los omega-3, actúa sobre la disminución del contenido de ácido araquidónico en las células del sistema inmunitario, este último con capacidad proinflamatoria. Esta simple acción ejerce un efecto estimulante sobre la reducción inflamatoria 6 .

Un dato importe que tener en cuenta es que este tipo de "grasas saludables» son esenciales, lo que significa que el cuerpo humano no tiene la capacidad para sintetizarlas, por lo que siempre deben ser aportadas mediante la alimentación. 


\section{NUTRIENTES CON POTENCIAL ANTIINFLAMATORIO}

Para realizar una dieta antiinflamatoria, basta con seguir unas simples indicaciones, asegurando el consumo de alimentos como:

- Pescado azul unas tres veces por semana, ya que es rico en ácidos grasos del tipo omega-

3. El arenque, la sardina, el salmón o el atún son los pescados azules que más fácilmente podremos encontrar.

- Verduras y frutas a diario, ya que son una fuente imprescindible de antioxidantes y de fibra. Estos alimentos también nos aseguran un aporte de micronutrientes con poder antiinflamatorio como el magnesio y el cinc, y de metabolitos como los flavonoides. Consumir cinco veces al día dichos alimentos nos asegurará una buena dosis de estos beneficiosos nutrientes.

- Frutos secos como las nueces, al menos, dos o tres veces por semana por su contenido en omega-3 y selenio, otro nutriente con propiedades antiinflamatorias.

- Aceite de oliva virgen a diario como aliño en lugar de aceites vegetales, salsas y otros aliños ricos en azúcares y grasas, también por su contenido en ácidos grasos precursores de los omega-3.

- Abrir la mente hacia el mundo de las especias nunca está de

\section{La dieta mediterránea, rica en verduras y fruta, cereales de grano entero, legumbres, aceite de oliva y pescado, se convierte en la opción más recomendada.}

más, ya que, aparte de ofrecernos una nueva forma de dar color y sabor a nuestros platos, también pueden resultar grandes aliados. La cúrcuma y el jengibre son, por ejemplo, unas de las especias con más fama antiinflamatoria.

Las ventajas de seguir estas directrices dietéticas no terminan aquí. Aparte de lograr reducir la inflamación, también se consigue rebajar las grasas y azúcares refinados en la alimentación, hecho que conlleva una reducción del peso corporal. Uno de los problemas asociados con mayor frecuencia al consumo de medicación antidepresiva es el sobrepeso, que, por otro lado, resulta ser uno de los efectos adversos que ocasionan un marcado malestar, tanto físico como emocional, entre los pacientes que lo padecen, aparte de ser un potencial riesgo para su salud cardiovascular.

Esta posible pérdida de peso y la mayor percepción de sensación de bienestar asociadas al seguimiento nutricional demuestran otro de los beneficios a favor de la revisión de la alimentación en el tratamiento.

Es por ello por lo que, como se indica al inicio de este artículo, cada vez más se aprecia una apertura hacia la visión multidisciplinaria del abordaje terapéutico de la depresión, dejando en manos de cada profesional la labor perteneciente a su competencia, abogando por el diálogo y la colaboración entre profesionales, siempre todo ello enfocado hacia el éxito del paciente en su recuperación.

\section{BIBLIOGRAFÍA}

1. Organización Mundial de la Salud. Depresión. 2017. Disponible en: http://www.who.int/mediacentre/ factsheets/fs369/es/

2. Southwick SM, Vythilingam $M$, Charney DS. The psychobiology of depression and resilience to stress: implications for prevention and treatment. Annu Rev Clin Psychol. 2005;1:255-91.

3. Duman RS. Neurotrophic factors and regulation of mood: role of exercise, diet and metabolism. Neurobiol Aging. 2005;26 Suppl 1:88-93.

4. Ontiveros M. Depresión y calidad de la dieta: revisión bibliográfica. Arch Medic. 2016;12:1-6.

5. Almond M. Depression and inflammation: examining the link. Curr Psychiatr. 2013;12(6):2432.

6. Rodrigo Valenzuela B, Tapia G, González M, Valenzuela A. Ácidos grasos omega-3 (EPA y DHA) y su aplicación en diversas situaciones clínicas. Rev Chil Nutr. 2011; 38(3):356-67. 